\title{
Soziale Arbeit und
}

"konsumspezifische Probleme «

\section{Eine semantisch-begriffliche Spurensuche nach dem Verbraucher}

\author{
Bernd Birgmeier und Martin Stummbaum
}

Soziale Arbeit hat es heute vielfach mit Menschen zu tun, die Ausprägungen konsumistischer Selbstbeeinträchtigungen, konsumbedingter Störungen sozialer Beziehungen oder inadäquater Bewegungen in Geld-und Tauschverhältnissen zeigen. Stichworte dafür sind Armut, Sucht, Überschuldung und »abweichendes Verhalten ". Trotz dieser Umstände hat sich die Soziale Arbeit bislang erfolgreich um den so wichtigen Faktor des Konsums gedrückt.

Die Ausblendung des Konsums aus der Sozialen Arbeit ist umso verwunderlicher, weil sie sich ja zunächst als eine Menschenwissenschaft verstehen will, die dementsprechend auch sämtliche (wenn auch nicht alle) Aspekte des MenschSeins zu berücksichtigen hat. Dass jedoch das Mensch-Sein in den gesellschaftlichen und soziokulturellen Bedingtheiten heute in erster Linie über den anthropologischen Grund- und Wesenszug des Verbrauchers (lat.: consumere; engl.: consumer) bestimmt wird, darüber hinaus der moderne Mensch ohne Konsum überhaupt nicht (mehr) überlebensfähig ist und wir daher auch der Tatsache ins Auge blicken müssen, dass ausnahmslos alle Menschen - auch und vor allem die Adressaten der Sozialen Arbeit - in den Konsumismus eingebunden sind und durch ihn in individuelle und soziale Problemlagen gedrängt werden können: diesen Faktizitäten wurden in der Sozialen Arbeit bis dato nur äußerst wenig Aufmerksamkeit geschenkt.

Konsumismus - so Hochstrasser - ist $\mathrm{zu}$ verstehen als eine Kultur, in welcher der Konsum der Menschen in ihrem alltäglichen Leben eine dominierende Stellung einnimmt und solcherart selbst ein charakteristischer Bestandteil der Kultur wird « (2008, 42). Zwar ermöglicht eine solche Kultur im Idealfall den Aufbau subjektiven Sinns und die Unterscheidung zwischen dem Ich und dem Anderen, falls die konsumistischen Möglichkeiten für selbst definierte und identitätsstiftende Zwecke genutzt werden; andererseits kann eine solche Kultur aber auch eine konsumistische Vereinnahmung der Subjekte bewirken, die dann den Diktaten des Markts hilflos ausgeliefert sind und so zum Spielball kapitalistisch-ökonomischer Interessen und »Konkurrenzveranstaltungen « werden (vgl. ebd. 44).

Problematisch - und zu einem wichtigen Thema für die Soziale Arbeit - wird der Konsumismus also dann, wenn die Grenzen eines "Normalkonsums « tangiert oder verletzt werden und sich beim Klienten Tendenzen oder Ausprägungen konsumistischer Selbstbeeinträchtigungen, konsumbedingter Störungen sozialer Beziehungen oder inadäquater Bewegungen in Geld- und Tauschverhältnissen zeigen. Dass ein Großteil sozialarbeiterischer und sozialpädagogischer Themen letztlich auf ein inadäquates Verhältnis des Einzelnen zum Konsum zurückzuführen ist, wir daher auch von "konsumspezifischen Problemen « in der Sozialen Arbeit sprechen müssen, wird spätestens dann deutlich, wenn wir den Blick beispielsweise auf Armut, Sucht, Überschuldung, Abweichung und ähnliches richten und nach den Bedingungen und Auslösern dieser Problematiken konkret im Konsumverhalten suchen (vgl. Hochstrasser 2008).

Es ist erschreckend, wie hoch die Korrelation zwischen fehl verstandenem und gestörtem Konsumismus und individuellen Problemen ist und dementsprechend auch: wie schwerwiegend sich die Folgen des Missbrauchs einer kapitalistisch orientierten Ökonomie für die Klienten der Sozialen Arbeit auswirken können. Grund genug, diejenigen Mechanismen aufzudecken, die die Dominanz konsumistischer Bedürfnisse erklären helfen und ökonomistische Missbrauchs-Szenarien zum Verbraucher aufdecken. Dazu ist es zunächst nötig, einen (konsumistischen) Blick auf die Rolle der Bedürfnisse im menschlichen Leben zu werfen, um 
hieraus auch die Vorgehensweisen und Wirkungen einer (kapitalistischen) Ökonomik zu verstehen. Anschließend sollen - im Rahmen einer Kritik am Modell des homo oeconomicus - Facetten zu Aspekten des »Missbrauchs « aufgedeckt werden, mit denen besonders die negativen Kehrseiten des Konsumismus deutlich werden und die gerade die Soziale Arbeit dazu zwingen, den »homo abusus « neu zu denken und verstärkt Programme zum Schutz vor dem Missbrauch von Verbrauchern zu entwickeln.

\section{Konsum als menschliches Grundbedürfnis?}

Besonders neuere neurowissenschaftliche Forschungen haben gezeigt, dass es neben den - altbekannten - physischen und physiologischen ebenso (für das Leben) wichtige psychische Grundbedürfnisse gibt. Diese sind zu verstehen als »Bedürfnisse, die bei allen Menschen vorhanden sind und deren Verletzung oder dauerhafte Nichtbefriedigung zu Schädigungen der psychischen Gesundheit und des Wohlbefindens führen " (Grawe 2004, 185). Dabei geht es also weniger um solche Grundbedürfnisse, mit denen das Überleben des »Biosystems Mensch « gewährleistet werden kann, sondern um solche, die das Bedürfnis nach

- Orientierung, Kontrolle und Kohärenz

- nach Bindung

- nach Selbstwerterhöhung und

- nach Lust thematisieren (vgl. ebd.).

Gerade das zuletzt genannte psychische Bedürfnis scheint bei der Annäherung an eine semantische Grundlegung zum Verbraucher eine wichtige Rolle zu spielen; denn hier werden - bewusst und unbewusst - die meisten biologischen Ursprünge zum Konsum gelegt; nicht zuletzt durch die Prinzipien des Lustgewinns und der Unlustvermeidung. Was also - so könnte sich der Konsument selbst fragen - bereitet mir Freude (und führt beim Erwerb darüber hinaus auch noch zu einer positiven Außenwirkung und hierüber zu einer sozial bedingten Selbstwerterhöhung) und - demgegenüber - was bzw. welche erwerbbaren Güter werfen eventuell ein negatives Bild auf mich? Mit diesen Aspekten sind emotionale Bewertungen von Konsumreizen verbunden, die demnach entweder zur Identifizierung oder zur Diskriminierung beitragen.
Es versteht sich von selbst, dass solche neuronalen Mechanismen in der Wirtschaft besonders von werbepsychologisch geschulten Marketing-Dienstleistern erkannt wurden und diese in regelmäßigen Abständen neue Trends und Moden produzieren, die den Verbraucher dazu motivieren sollen, dem - will man up to date sein und den Zug der Zeit nicht verpassen - Mainstream zu huldigen und dementsprechende Güter zu konsumieren. Besonders bei Kindern und Jugendlichen treffen solche Marketing-Taktiken bisweilen mitten ins Schwarze, so dass das kontinuierliche Herunterladen von neuesten Handy-Melodien, der Konsum hipper Musik (dargeboten in Fernsehkanälen wie VIVA oder MTV), Outfits, technischer Geräte (wie Playstations etc.), Jugendzeitschriften offensichtlich normal geworden ist. In diesem Zusammenhang sprechen Experten auch von einer »kommerzialisierten « Jugendkultur, die sich spätestens in den 1990-er Jahren deutlich herauskristallisierte und in der besonders der materielle Habitus von Kindern und Jugendlichen (über-) betont wird.

Nicht von ungefähr wird daher auch die Wirtschaft als ein »System von Bedürfnissen « (Weber) definiert, das - als Subsystem der Macht (Parsons; Weber) für die Beschaffung von Konsumgütern verantwortlich ist und dort »wirtschaftet «, wo die Mittel für die Befriedigung ganz verschiedener Bedürfnisse mobilisiert werden (vgl. Berger 1998).

\section{Der homo oeconomicus als homo abusus}

Das Konsumieren - als Bedürfnis und vielleicht wichtigste Form des Habens in den heutigen »Überflussgesellschaften «offenbart jedoch ein janusköpfiges Gesicht: zum einen vermindert Konsum die Angst vor Besitzlosigkeit (des Konsumgutes); andererseits zwingt er den Menschen dazu, immer mehr zu konsumieren, denn die Konsumgüter können - nach den Gesetzen der Trendforschung - an Relevanz und Wichtigkeit verlieren. Ein Konsument - so Fromm - ist demnach der »ewige Säugling, der nach der Flasche schreit « $(1982,37)$ und beginnt sich mit der Formel zu identifizieren: »Ich bin, was ich habe und was ich konsumiere" (1982, 37).
Diejenige Disziplin, die besonders den Konsumenten und das, was er hat (bzw. unbedingt braucht!), ins Auge fasst, ist die Ökonomik. Als Sozialwissenschaft gibt sie - in klassischer (national-) ökonomischer Tradition von Smith über v. Hayek bis Buchanan - vor, ihren »letzten Sinn « in der Gestaltung der sozialen Welt und ihrer sozialen Ordnung gefunden zu haben, angefangen vom einmaligen Tausch bis zum Zusammenleben von Menschen in (unterschiedlichen sozialen Gruppen) der Gesellschaft. Dabei geht sie von einem Menschenbild aus, mit dem ein konsumistisches Haben als Sein postuliert wird und das im "homo oeconomicus « einer neokapitalistisch durchdrungenen Lebensform Rechnung trägt, die das Individuum dazu verpflichtet, zielorientiert seinen persönlichen Nutzen zu maximieren.

Mit der aus diesem Menschenbild resultierenden ökonomischen Grundformel, nämlich: »Individuen maximieren ihren Nutzen unter Restriktionen « (Homann/Suchanek 2000, 29), rücken zwei auch zum Verständnis der Ursachen falschen Konsumverhaltens - wichtige ökonomische Grundbegriffe in den Vordergrund. Einmal der Nutzen, den der Verbraucher durch situationsbedingte, handlungsbestimmende Vorteilserwartungen in Abhängigkeit von seiner sozialen Umwelt und seiner biografischen Situation erkennt und die ihm letztlich - gekoppelt an die Anreize, die der Markt als »aufmerksamkeitslenkende Maßnahmen « (wie z. B. Werbung) initiiert - als Gründe und Motive dienen, sich konsumistisch zu verhalten. Zum anderen die Restriktionen, die für die Nutzenmaximierung des Konsumentenverhaltens eine eingrenzende Bedeutung haben und den Wünschen des Individuums und der Befriedigung dessen Bedürfnisse Grenzen setzen (vgl. ebd. 2000, 31). Nützlich ist ein Konsumverhalten also dann, wenn sich der Verbraucher durch den Erwerb konsumistischer Mittel die Befriedigung eines auf Wünschen beruhenden Bedürfnisses verspricht; gleichzeitig steht die Befriedigung dieser Bedürfnisse jedoch in der Gefahr, wenn der Konsument innerhalb seines nach Zielen strebenden und an individuellen Motiven orientierten Konsumierens erkennen muss, dass seine individuellen Mittel und Ressourcen möglicherweise nicht vorhanden oder zu knapp sind. 


\section{Der Verbraucher im Zeichen des Scheiterns}

Besonders die von Armut betroffenen Klienten der Sozialen Arbeit müssen die Frustrationen ertragen, die sich aufgrund der Unmöglichkeit eigener Bedürfnisbefriedigung wegen fehlender Mittel und Ressourcen einstellen. Sie finden sich gefangen in der Polarität zwischen dem öffentlichen und ökonomischen Diktat, mit dem eine zeitgerechte Teilhabe und Teilnahme an Gesellschaft und Kultur überhaupt erst möglich zu sein scheint, und dem Erleben und Erkennen eines Daseins, das - wenn wir hier noch einmal Fromm bemühen dürfen - eigentlich gar nicht da ist, weil es nichts hat ... obwohl es so vieles bräuchte! Der Begriff »brauchen « - so Tillmann - drückt daher immer auch die »Beziehung zu einem anderen oder etwas anderem aus. Irgendwer oder irgendwas wird gebraucht. Verbrauch und Missbrauch bezeichnen daher verfehlte Beziehungen zu Gegenständen, Sachverhalten oder Menschen, aber auch alle Fälle, wo Entfaltung behindert, Wachstum unterdrückt und Selbstbestimmung reguliert wird «, weil jeder Mensch auch ein Scheiternder, ein sich Verfehlender ist (vgl. Tillmann 1994, 66 f.).

Mit diesen Gedanken lassen sich weitere »Spuren« erkennen, mit denen der Verbraucher verstärkt ins Blickfeld des Interesses der Sozialen Arbeit zu rücken ist. Bisher haben wir den Verbraucher als Konsumenten identifiziert, für den - im Normalfall - der Konsum eine positive, in konsumistischen Grenzfällen und aufgrund kapitalistisch-ökonomischer Diktate jedoch auch eine Reihe an negativen Erscheinungen zeitigt. Mit dem - insbesondere für die Soziale Arbeit - notwendigen Fokus auf diese negative Kehrseite stellt sich nun konkret die Frage nach dem Missbrauch des Konsums und darüber nach dem durch den in diesem Falle vom Konsum(ismus) missbrauchten Menschen. In diesen heuristischen Kontext passt vor allem auch der »homo abusus «, den Tillmann (1994; 2007) in seiner umfassenden Bedeutung versteht als den verbrauchten, missbrauchten Menschen, den verbrauchenden, missbrauchenden und den sich verbrauchenden, sich missbrauchenden Menschen; kategorisiert als: der ausgebeutete, ausbeutende, sich ausbeutende Mensch, der überforderte, überfordernde, sich überfordernde
Mensch, der ungeschützte, Schutz zerstörende, sich nicht schützende Mensch und der ungehaltene, Halt zerstörende, sich Halt zerstörende Mensch.

Mit diesen anthropologischen Vorannahmen zum missbrauchten Menschen wäre die Soziale Arbeit imstande, neue Zusammenhänge und Abhängigkeiten zum Konsumismus zu beschreiben und an einer für sie typischen Betrachtungsweise des menschlichen Verhaltens anzusetzen; beispielsweise am "Missbrauch « des Verbrauchers, der - in Gestalt des homo disoeconomicus - einerseits von den Mächten und Kräften einer kapitalistisch-ökonomischen Logik missbraucht und ausgebeutet wird, andererseits sich jedoch auch selbst missbraucht, in dem er entweder seine Identitätsfindung durch eine zu starke Orientierung am Konsumismus festschreibt oder seine Mittel und Möglichkeiten als Verbraucher überschätzt. Der Konsumismus erschafft dadurch ein gesellschaftliches Idealbild, mit dem der Begriff des »Brauchens « kapitalisiert, konterkariert und dementsprechend auch entmoralisiert wird - dies mit der Folge, dass der Mensch, will er seine Menschlichkeit nicht verfehlen, zu einem Wesen zu mutieren droht, das rein an konsumistischen Maßgaben orientiert sein muss: der am homo oeconomicus scheiternde "homo abusus « (Tillmann 1994, 69; 2007, 79 ff.)!

\section{Zum Schutz des Missbrauchs des Verbrauchers - ein Fazit}

Solange die Ökonomik ihre Modellierungen des menschlichen Verhaltens als das » Maß aller Dinge « behauptet, wird das Scheitern zu einem humanistischen (?) Programm ökonomischer Imperialismen, mit denen nicht nur dem Sozialdarwinismus eine Renaissance beschert wird, sondern auch allen »Subjektivierungsformen « (Bröckling 2007), die das Soziale, das Gemeinschaftliche und das Miteinander allmählich zerstören.

Wichtig ist es deshalb, die - altbewährte und an eine humanistische Moral gebundene - Lebensweltorientierung in der Sozialen Arbeit nicht für eine konsumistische Ökonomisierung zu opfern und dementsprechend auch die Subjekte nicht in den Rahmen einer ökonomisch geprägten instrumentellen oder strategischen Rationalität zu stellen, mit der der
Mensch instrumentalisiert und seiner Grund- und Menschenrechte beraubt wird.

Daher wird es in Zukunft notwendig werden, das wirklich »Soziale « in der Marktwirtschaft wieder zu beleben und das individuelle und soziale Sein jedes einzelnen Bürgers als »Humankapital « anzuerkennen, das sich - gottlob! - mit materiellen Mitteln nicht konsumieren lässt! Nur so ist der über Konsumklima-Indexe messbare "Verwertungsprozess « des Menschen zu stoppen. Etwas mag es da schon helfen, das Thema Verbraucherschutz - wofür es in unserem Staat sogar ein eigenes Ministerium gibt! - über Problempunkte wie Gentechnik, Spielzeugsicherheit, Pestizitbelastungen oder das Gammelfleisch hinaus zu denken ...

\section{Literatur}

Berger, J. (1998): Wirtschaftssystem. In: Schäfer, B./Zapf, W. (Hg.): Handwörterbuch zur Gesellschaft Deutschlands. Opladen. S. 710-719.

Bröckling, U. (2007): Das unternehmerische Selbst. Frankfurt am Main.

Fromm, E. (1982): Haben oder Sein. München.

Grawe, K. (2004): Neuropsychotherapie. Göttingen.

Hochstrasser, F. (2008): Zusammenhänge zwischen Konsumismus und Sozialer Arbeit. In: neue praxis 1/2008. S. 42-57.

Homann, K./Suchanek, A. (2000): Ökonomik. Eine Einführung. Tübingen.

Tillman, J. (1994): Der Gegenstand der Sozialarbeitswissenschaft. In: EFH Hannover (Hg.): Annäherung an eine Sozialarbeitswissenschaft Hannover. S. $65-76$.

Tillmann, J. (2007): Trajektivität. Hannover. 